\title{
Exploring the Effect a Speaker Series has on Students Level of Multicultural Sensitivity and Cultural Competence Awareness
}

\author{
Christine Karpinski \\ West Chester University, ckarpinski@wcupa.edu \\ Scott Heinerichs \\ West Chester University
}

Follow this and additional works at: https://nsuworks.nova.edu/ijahsp

Part of the Bilingual, Multilingual, and Multicultural Education Commons, Curriculum and Instruction Commons, and the Medicine and Health Sciences Commons

\section{Recommended Citation}

Karpinski C, Heinerichs S. Exploring the Effect a Speaker Series has on Students Level of Multicultural Sensitivity and Cultural Competence Awareness. The Internet Journal of Allied Health Sciences and Practice. 2015 Jul 01;13(3), Article 6.

This Manuscript is brought to you for free and open access by the College of Health Care Sciences at NSUWorks. It has been accepted for inclusion in Internet Journal of Allied Health Sciences and Practice by an authorized editor of NSUWorks. For more information, please contact nsuworks@nova.edu. 


\title{
Exploring the Effect a Speaker Series has on Students Level of Multicultural Sensitivity and Cultural Competence Awareness
}

\begin{abstract}
Purpose: The population of the United States continues to become more diverse each day, and this changing demographic impacts our healthcare system, demanding that healthcare providers begin to provide culturally competent services. In order to have culturally competent practitioners, it would be prudent to incorporate the concepts of cultural competence effectively into respective health professions curricula. The purpose of this study was to assess the effectiveness of a three-part, semester-long cultural competency speaker series on students' level of multicultural sensitivity and their attitudes toward cultural competence and its effect on healthcare. Methods: A convenience sample of 118 athletic training and nutrition undergraduate students participated in this study through their attendance at three 90-minute lectures over the course of the fall 2012 semester. A pre/post questionnaire study design was utilized. Two valid and reliable surveys, the Multicultural Sensitivity Scale (MSS) and the Health Beliefs Attitudes Survey (HBAS) were adapted from the literature. The MSS was used to measure students' level of multicultural sensitivity and the HBAS was used to assess their attitudes on how cultural competence affects health care quality. Each survey was completed by subjects prior to and at the conclusion of the speaker series. Results: There was a significant difference between the mean total score of the HBAS prior to and after the speaker series $(t=4.01 ; p$
\end{abstract}




\title{
TISAHSP
}

\section{The Internet Journal of Allied Health Sciences and Practice}

Dedicated to allied health professional practice and education

Vol. 13 No. 3 ISSN 1540-580X

\section{Exploring the Effect a Speaker Series has on Students Level of Multicultural Sensitivity and Cultural Competence Awareness}

\author{
Christine Karpinski, PhD, RD, CSSD, LDN1 \\ Scott Heinerichs, EdD, ATC2
}

1. Assistant Professor, Department of Nutrition, West Chester University, West Chester PA

2. Associate Professor, Department of Sports Medicine, West Chester University, West Chester PA

United States

\begin{abstract}
Purpose: The population of the United States continues to become more diverse each day, and this changing demographic impacts our healthcare system, demanding that healthcare providers begin to provide culturally competent services. In order to have culturally competent practitioners, it would be prudent to incorporate the concepts of cultural competence effectively into respective health professions curricula. The purpose of this study was to assess the effectiveness of a three-part, semester-long cultural competency speaker series on students' level of multicultural sensitivity and their attitudes toward cultural competence and its effect on healthcare. Methods: A convenience sample of 118 athletic training and nutrition undergraduate students participated in this study through their attendance at three 90-minute lectures over the course of the fall 2012 semester. A pre/post questionnaire study design was utilized. Two valid and reliable surveys, the Multicultural Sensitivity Scale (MSS) and the Health Beliefs Attitudes Survey (HBAS) were adapted from the literature. The MSS was used to measure students' level of multicultural sensitivity and the HBAS was used to assess their attitudes on how cultural competence affects health care quality. Each survey was completed by subjects prior to and at the conclusion of the speaker series. Results: There was a significant difference between the mean total score of the HBAS prior to and after the speaker series $(t=4.01 ; p<.001)$; however, no significant difference was observed between the mean total score of the MSS prior to and after the series $(t=0.54 ; p=.59)$. The mean total HBAS score prior to and after the series was $71.8 \pm 5.9$ and $76.8 \pm 6.0$, respectively ( $2.1 \%$ increase). The mean total MSS score prior to and after the series was $83.1 \pm 13.3$ and $82.5 \pm 13.3$ (1\% decrease). Conclusions: The speaker series significantly changed the students' attitudes related to health care quality and multicultural sensitivity and suggested that it may have broadened the student's level of multicultural sensitivity. Future research needs to focus on the most effective duration and delivery of cultural competency training to health professionals.
\end{abstract}

\section{INTRODUCTION}

The population of the United States continues to become more diverse each day. Demographic trends in the United States project that by 2050, the proportion of non-Hispanic whites in the population will decrease to approximately 50 percent. ${ }^{1}$ These changing demographics have impacted our healthcare system, demanding that health care providers begin to practice cultural competence. Additionally, specialized accrediting bodies of health professions have incorporated competencies related to cultural competence in their respective accreditation standards. 2,3

Culture competence is a complex concept that has four components: 1) Awareness of one's own cultural worldview, 2) Attitude towards cultural differences, 3) Knowledge of different cultural practices and worldviews, and 4) Cross-cultural skills. Developing cultural competence results in an ability to understand, communicate with, and effectively interact with people across cultures. ${ }^{4}$ The first component of awareness has been termed multicultural sensitivity. Specifically, multicultural sensitivity is defined as increasing awareness of one's own and other's culture. ${ }^{5}$ There have been a variety of ways in which health professions have implemented and evaluated cultural competence in education programs. Specific strategies that have been successful in the literature include teaching motivational techniques to students, utilizing computer simulation, and immersing in international community service experiences. ${ }^{6-8}$ Although there have been successful approaches, it is also evident that cultural competence

(c) The Internet Journal of Allied Health Sciences and Practice, 2015 
is a difficult area to address with students. One reason for this is a faculty member's reluctance to feel comfortable given the ever-changing content as it pertains to racial and ethnic minorities. Unless this is an area of scholarly expertise for the faculty member, the need to stay up-to-date for teaching this material is lacking in most academic settings. ${ }^{9}$ Thus, a lack of professional development or expertise in this area forces many programs to focus on basic cultural knowledge with limited application or experiential opportunities for students to develop intercultural communication skills and evaluate ways to impact their value and belief systems. ${ }^{10}$

It is important for health programs to identify and implement appropriate methods to teach and assess students' cultural competence to ensure knowledge can be retained and applied in the future. We recognized the deterrents discussed above within our programs and wanted to explore innovative ways to teach and assess cultural competence with our students. Specifically, we wanted to focus on the first component of multicultural sensitivity. Basic concepts of multicultural sensitivity were already being addressed within our coursework. In addition, all students on our campus take a general education course focused on marginalized groups in society. However, our programs were lacking in how to incorporate the expert opinions of individuals well versed in multicultural sensitivity within health disciplines. Being able to add this component within our programs would help students increase awareness in a more discipline specific context and possibly influence the quality of care they will provide as future healthcare professionals. Therefore, the purpose of this article is to assess students' level of multicultural sensitivity and their attitudes on how cultural competence relates to quality of healthcare after a semester-long speaker series.

\section{METHODS \\ Participants}

A convenience sample of 118 undergraduate third- and fourth-year Athletic Training or Nutrition majors (students) were recruited for this study. As part of a classroom assignment, students were encouraged to attend three 90-minute lectures held monthly during the semester as part of a cultural competence speaker series organized by the authors. Outside speakers were recruited from three health disciplines (athletic training, nursing, and nutrition) for the speaker series and provided with a suggested structure for their presentation. Each of the speakers were practitioners within their field, as well as recognized scholars (e.g., had peer reviewed publications and national/regional presentations) in the area of cultural competence. Additionally, each speaker focused their talk on a different culture so that students could better understand various populations they are likely to encounter in the future. The athletic training expert addressed people of color, the nursing expert addressed lesbian, gay, bisexual, and transsexual individuals, and the nutrition expert addressed individuals with disabilities, such as vision and hearing impaired.

A pre-posttest design was utilized for this study. All participants completed two valid and reliable surveys--the Health Beliefs Attitudes Scale (HBAS) and the Multicultural Sensitivity Scale (MSS)--prior to and at the conclusion of the speaker series. The Health Beliefs Attitudes Survey (HBAS) was used to assess the impact the speaker series had on students' attitudes toward how cultural competency relates to healthcare quality. ${ }^{11}$ The HBAS centers on four constructs related to determining the importance of 1) Opinion - Importance of assessing patients' perspectives and opinions (statements 1,3,6, 10,13); 2) Belief - Importance of determining patients' beliefs for history taking and treatment (statements 2, 4, 8, 11); 3) Context - Importance of assessing patients' psychosocial and cultural contexts (statements 9, 12, 14); and 4) Quality - Importance of knowing the patient's perspective for providing good health care (statements $5,7,15$ ). The HBAS consists of 15 items, scored on a 6-point Likert-type scale. The research protocol was approved by the Institutional Review Board.

In addition to understanding student's attitudes toward cultural competency and health care quality, it was equally important to have an understanding of the impact of the speaker series on increasing students' awareness of their own and other's cultures (i.e. multicultural sensitivity). Jibaja-Ruth et al validated the Multicultural Sensitivity Scale (MSS) instrument to measure multicultural sensitivity between teachers and students (K-12).12 This tool was later adapted and utilized with physician assistant students to show an increase in their level of multicultural sensitivity following coursework devoted to the topic of cultural competence. ${ }^{13}$ The Multicultural Sensitivity Scale (MSS) consists of 18 items, scored on a 6-point Likert-type scale.

\section{Data Collection and Analysis}

Data were collected from all participants prior to the first speaker and again at the conclusion of the speaker series. A paired ttest was used to assess the effectiveness the speaker series had on the students' attitudes as it pertains to cultural competence affecting health care quality (HBAS) and their level of multi-cultural sensitivity (MSS) and by comparing the mean score of each survey before and after the speaker series. Paired t-tests were also calculated for the mean scores of each individual statement prior to and after the speaker series.

(c) The Internet Journal of Allied Health Sciences and Practice, 2015 


\section{RESULTS}

The sample of 118 students was predominately white $(n=111,94.1 \%)$, female $(n=91,77.1 \%)$, juniors $(n=32,27.1 \%)$ and seniors $(n=86,72.9 \%)$. Fifty-three percent $(n=63)$ were nutrition majors and $46.6 \%(n=55)$ were athletic training majors. The majority of students $(n=83,70.3 \%)$ reported zero semesters of clinical experience prior to fall 2012. Half reported holding at least one job related to their chosen profession. More than half $(n=81,68.6 \%)$ reported taking a diversity-themed course. The majority of students $(n=76,64.4 \%)$ attended all three lectures, while $27(22.9 \%)$ attended two lectures, $12(10.2 \%)$ attended one, and 2 did not attend. At baseline, nutrition majors had a significantly higher HBAS scores than athletic training majors $(t=2.93 ; p=.009)$. After the lecture series, nutrition majors had a significantly higher HBAS scores than athletic training majors $(t=3.01 ; p=.005)$.

There was a significant difference between the mean total score of the HBAS prior to and after the speaker series ( $t=4.01$; $p<.001$ ); however, no significant difference was observed between the mean total score of the MSS prior to and after the series ( $t$ $=0.54 ; p=.59)$. The mean total HBAS score prior to and after the series was $71.8 \pm 5.9$ and $76.8 \pm 6.0$, respectively $(2.1 \%$ increase). The mean total MSS score prior to and after the series was $83.1 \pm 13.3$ and $82.5 \pm 13.3$ ( $1 \%$ decrease).

The opinion, context, and quality constructs were significantly different before and after the lecture series, whereas the belief construct was not significantly different (Table). Statements $6(5.10 \pm .75), 9(5.21 \pm .81)$, and 12 (5.36 \pm .70$)$ had the highest level of agreement among the students prior to the lecture series, although statements 6 and 12 were not significantly different after the series. $(t=1.81 ; p=.07$ and $t=.98 ; p=.33$, respectively). After the lecture series, statements $9(5.53 \pm .68)$ and $12(5.52 \pm$

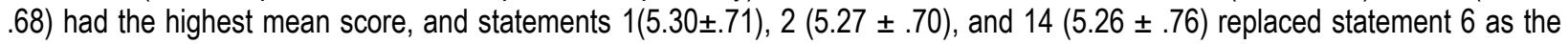
third highest mean score. The statements with the lowest mean score before and after the lecture series were statements 5 (3.02 $\pm .65), 7(3.49 \pm 1.5)$, and $15(3.53 \pm 1.4)$. After the lecture series, the same three statements had the lowest mean scores (statements $5(3.37 \pm .1 .5), 7(3.75 \pm 1.5)$, and $15(3.94 \pm 1.5)$.

Table 1. Health Beliefs Attitudes Survey (HBAS) Component Scores of Sample $(n=118)$

\begin{tabular}{|l|r|r|r|r|r|c|}
\hline & \multicolumn{2}{|c|}{ Pre-Intervention } & \multicolumn{2}{c|}{ Post-Intervention } & & \\
\hline \multicolumn{1}{|c|}{ Construct } & Mean & \multicolumn{1}{c|}{ SD } & Mean & \multicolumn{1}{c|}{ SD } & \multicolumn{1}{c|}{ t statistic } & P value \\
\hline Opinion ${ }^{1}$ & 4.87 & .53 & 5.18 & .51 & 3.72 & $<.001^{* *}$ \\
\hline Belief $^{2}$ & 5.04 & .44 & 5.23 & .46 & 1.57 & .13 \\
\hline Context $^{3}$ & 5.23 & .56 & 5.51 & .56 & 4.62 & $<.001^{\text {** }}$ \\
\hline Quality & 4 & 1.15 & 3.70 & 1.15 & 3.82 & $<.001^{* *}$ \\
\hline
\end{tabular}

** $p<.001$

Note: SD-standard deviation; Component scores are the mean of responses to items scored on a 6-point scale (1=strongly disagree to $6=$ strongly agree)

1 Importance of assessing patients' perspectives and opinions (statements 1,3,6,10,13)

2 Importance of determining patients' beliefs for history taking and treatment (statements $(2,4,8,11)$

3 Importance of assessing patients' psychosocial and cultural contexts (statements $9,12,14$ )

${ }^{4}$ Importance of knowing the patient's perspective for providing good health care (statements $5,7,15$ )

\section{DISCUSSION}

Cuellaer et al found that cultural competence is a difficult subject topic for faculty to teach because of their limited expertise within this area and the changing ethnic demographics in our society. ${ }^{9}$ Our study provided an alternative method for programs to consider as it relates to teaching and assessing one aspect of cultural competence, which is multicultural sensitivity within health programs. Specifically, we wanted to see if there was in an improvement in the students' level of multicultural sensitivity and attitudes on how cultural competence affects health care quality following a semester-long speaker series presented by scholars and clinicians from health disciplines. Guest speakers have been demonstrated to enhance the educational experience of students because they provide unique insights and perspectives from their fields. ${ }^{14}$ Additionally, we chose individuals who not only could provide perspective from their field, but were also accomplished scholars in each of the cultural domains discussed.

The opportunity for students to hear from both scholars and practitioners proved to be valuable, as there was a positive change in students' attitudes of how cultural competence can affect healthcare quality. The HBAS centers on four constructs related to determining the importance of assessing patients' perspectives and opinions (opinion), determining patient beliefs for history taking and treatment (belief), assessing patients' psychosocial and cultural contexts (context), and knowing the patient's perspective for providing good health care (quality). Though we could have constructed lectures and assignments for our students to engage in to evaluate these areas, it would not have been as authentic. Utilizing clinicians and scholars of cultural competence enabled the students to better understand each of these four areas because the learning environment created for the students was more genuine. The speakers were able to provide specific context and bring real world issues as they relate to

(C) The Internet Journal of Allied Health Sciences and Practice, 2015 
multicultural sensitivity to our students using their experiences as well as research. ${ }^{15}$ Guest speakers have been shown to improve students' recognition and awareness of cultural and gender diversity. ${ }^{14,16-18}$

Some comparisons can be made between this study and that of Crosson et al. ${ }^{17}$ Although the sample characteristics (medical students and undergraduate nutrition and athletic training students) and delivery methods (lecture series versus course curriculum) were somewhat different, it is interesting to compare the results of the HBAS. This study reported significantly different mean scores for the opinion, context, and quality constructs, but not belief. Crosson et al reported significantly different mean scores for the opinion and belief constructs, but not context or quality among first-year medical students. ${ }^{17}$ They posited that their intervention focused on teaching students how to assess patients' perspectives and did not necessarily discuss what constitutes quality healthcare. Many of our students may not understand the relationship between asking the patients' about their cultural beliefs and making a diagnosis (belief). In addition, our students were told throughout the lecture series that the quality of health care depends on knowing the patients' perspectives (quality), and that was reflected in their post-series responses.

The individual statements with the highest mean score prior to the lecture series were 6, 9, and 12. Agreement for statement 9, however, significantly increased after the lecture series. Statement 9 read, "Healthcare professionals should learn about their patients' cultural perspective." Although agreement for statement 9 was already high prior to the lecture series, results suggest that the lecture series further supported the students' original understanding of psychosocial and cultural contexts. This is a concept that is not as apparent as the illness itself, which is what statements 6 and 12 focused on. The mean score for statements 6 and 12 on the HBAS were not significantly different before and after the lecture series $(p=.33$ and $p=.07$, respectively), but this could be attributed to the high mean score for these two statements prior to the lecture series. Although statement 6 remained high after the lecture series, statements 1,2, and 14 surpassed the mean score for statement 6 . Each of these statements represents a different construct (opinion, belief, and context, respectively). This suggests that the lecture series broadened the students' level of cultural competency. Although the mean score for the Quality construct significantly increased after the lecture series, statements 5,7 , and 9 remained the lowest mean scores both prior to and after the series. These three statements posited that knowing the patient's perspective is important in providing quality health care. This may suggest that although the students recognized the impact of culture on a patient's experience, they still believe that excellent healthcare can be provided without accounting for cultural perspectives. This attitude may have existed because the students attributed high quality healthcare with medical outcomes and did not account for interpersonal care. This survey did not assess medical outcomes, focusing instead on interpersonal healthcare or patient-centered care, which is defined by the IOM as "Providing care that is respectful of and responsive to individual patient preferences, needs, and values, and ensuring that patient values guide all clinical decisions."18

The ability of the speakers to use their experience as clinicians as well as their scholarly activity enabled our students to gain a more evidence-based perspective of what it means to be a culturally competent healthcare provider because the speaker's experiences were supported by direct claims from the literature. Though the series did impact students' attitudes toward how cultural competency relates to healthcare quality, the series did not result in a significant change in students' level of multicultural sensitivity. The MSS was used to assess students' level of multicultural sensitivity. It is not surprising that the speaker series was unable to demonstrate a change in students' level of sensitivity. Changes in sensitivity levels of students take time and the speaker series provided only a brief exposure to this topic. 13,17 It has been found that observable change can occur when students are involved in active learning types of activities such as clinical experiences that involve direct patient interactions, in addition to lectures. However, it was important to administer the MSS because it provided us with a baseline understanding of our students' level of sensitivity as it relates to cultural competence and enabled us to better interpret the findings of the HBAS.

Although we found a significant change in students' attitudes related to health care quality and cultural competence, we do not know the extent to which their attitudes will remain over time. Beagan assessed medical students attitudes three years after a pre-clinical course that discussed multicultural sensitivity and found no change. ${ }^{20}$ As programs begin to look at ways to assess and document their students' learning related to cultural competence, it would be important to follow up with cohorts of students at the end of their program to document changes in their attitudes and knowledge related to multicultural sensitivity to better understand what contributes to their learning (i.e. internships, course work, service learning, or other course work).

\section{CONCLUSION}

The findings of this study provided an assessment of a speaker series on the students' level of multicultural sensitivity and attitudes on how cultural competence affects health care quality. Providing a speaker series featuring practitioners who are also scholars in the area of cultural competence provides a unique opportunity for health programs to teach cultural competency and enhance the cultural competency curriculum. We believe the interdisciplinary approach of this series provided another advantage to the students, as they will most likely need to collaborate across healthcare professions.

(c) The Internet Journal of Allied Health Sciences and Practice, 2015 
Future research needs to focus on the most effective duration and delivery of cultural competency training to undergraduate health-related majors. The ultimate goal for programs is to move from the assessment of students' understanding and awareness of cultural beliefs and perspectives to the provision of culturally competent services.

\section{REFERENCES}

1. US Census Bureau. U.S. Interim Projections by Age, Sex, Race, and Hispanic Origin: 2000-2050. http://www.census.gov/population/www/projections/usinterimprojl. Accessed June 11, 2014.

2. Accreditation Council for Education in Nutrition and Dietetics. ACEND Accreditation Standards for Didactic Programs in Nutrition \&Dietetics Leading to Supervised Practice. Updated July 31, 2013 Version 1.04 of the 2008 DPD Standards. Academy of Nutrition and Dietetics. Chicago, IL. http://www.eatright.org/ACEND/. Accessed June 11, 2014.

3. Commission on Accreditation of Athletic Training Education. Updated 2011, $5^{\text {th }}$ Edition of the Athletic Training Education Competencies. http://www.nata.org/sites/defaultffiles/5th_Edition_Competencies.pdf. Accessed June 11, 2014.

4. Campinha-Bacote J. The Process of Cultural Competence in the Delivery of Healthcare Services: A Model of Care. $J$ Transcult Nurs. 2002;13(3):181-4. [PMID 12113146]

5. Government Printing Office: Cultural competence for evaluators: A guide for alcohol and other drug abuse prevention practitioners working with ethnic/racial communities. Washington, DC. DHHS -ADM-92-1884. 1992.

6. Salas-Lopez D, Soto-Greene M, Mouzon DM, et al. Evaluation of a cultural competency curriculum in the third year medicine clerkship. J Assoc Acad Minor Phys. 2006;17:54-60

7. Rutledge CM, Barham P, Wiles L, Benjamin RS, Eaton P, Palmer K. Integrative simulation: a novel approach to educating culturally competent nurses. Contemp Nurs. 2008;28(1-2):119-28. [PMID 18844565]

8. Dupre AM, Goodgold S. Development of physical therapy student cultural competency through international community service. J Cult Divers. 2007;14(3):126-34. [PMID 18314814]

9. Cuellar NG, Brennan AM, Vito K, de Leon Siantz ML. Cultural competence in the undergraduate nursing curriculum. J Prof Nurs. 2008;24(3):143-9. [PMID 18504027]

10. Knoblock-Hahn A, Scharff E, Elliot M. Cultural competence in dietetics education. J Am Diet Assoc. 2009;109(suppl 3):A12.

11. Dobbie AE, Medrano M, Olney C, Welsh K, Tysinger J. A curriculum in health beliefs for first-year medical students. Presented at the 2002 Society of Teachers of Family Medicine Predoctoral Education Conference in Tampa, Fla.

12. Jibaja-Rusth ML, Kingery PM, Holcomb JD, Buckner WP, Pruitt BE. Development of a Multicultural Sensitivity Scale. J Heal Educ. 1994;25(6):350-7.

13. Schneider V, Thompson BM, Celis MI. Multicultural sensitivity of physician assistant students after instituting cultural competence instruction. Persp Phys Asst Educ. 2005;16(2):103-6.

14. Murray G, Bollinger D. Developing cross-cultural awareness: Learning through the experience of others. TESL Can J 2001;19:62-72.

15. Glenwick $D$, Chabot $D$. The undergraduate clinical child psychology course: Bringing students to the real world and the real world to the students. Teach Psychol. 1991;18(1):21-4.

16. Butler R. Using gender balance to enhance teaching effectiveness. Bus Comm Q.1997;60(3):93-100.

17. Crosson JC, Deng W, Brazeau C, Boyd L, Soto-Greene M. Evaluating the effect of cultural competency training on

18. medical students attitudes. Fam Med. 2004;36(3):199-203. [PMID 14999577]

19. Nourse ME. Tapping campus talent for the collegiate business communication course. Bus Commun Q.1995;58(2):25-8.

20. Institutes of Medicine. National Academies of Medicine. Crossing the Quality Chasm: A New Health System for the 21st Century. Retrieved from https://www.iom.edu/ /media/Files/Report\%20Files/2001/Crossing-the-QualityChasm/Quality\%20Chasm\%202001\%20\%20report\%20brief.pdf. Accessed August 22, 2014.

21. Beagan BL. Teaching social and cultural awareness to medical students: It's all very nice to talk about it in theory, but ultimately it makes no difference. Acad Med. 2003;78(6):605-14. [PMID 12805041] 